\title{
Association of Sympathovagal Imbalance with Cognitive Deficit, Insulin Resistance and Oxidative Stress in Newly Diagnosed Hypertension
}

\author{
Subathra Thiruchengodu Ammaiyappan ${ }^{1}$, Gopal Krushna Pal ${ }^{1 *}$, Dhanalakshmi Yerrabelli ${ }^{1}$, Pravati Pal ${ }^{1}$, Nivedita \\ $\mathrm{Nanda}^{2}$
}

\section{Subathra Thiruchengodu Ammaiyappan 1 , Gopal Krushna Pal ${ }^{*}$, Dhanalak- shmi Yerrabelli' ${ }^{1}$, Pravati $\mathrm{Pal}^{1}$, Nivedita Nanda ${ }^{2}$}

\section{'Department of Physiology, Jawaharlal Institute of Post-graduate Medical education and Research (JIPMER), Puducherry - 605 006, INDIA. 2Department of Biochemistry, Jawaharlal Institute of Post-graduate Medical education and Research (JIPMER), Puducherry - 605 006, INDIA. \\ Correspondence \\ Dr. Gopal Krushna Pal \\ Dean, JIPMER, Karaikal (Puducherry) and Professor of Physiology, JIPMER, Puducherry - 605006 , INDIA \\ Phone: 0-91- 9344291160 \\ Email: drgkpa|@gmail.com \\ History \\ - Received: 13-07-2018 \\ - Revised: 20-09-2018; \\ - Accepted: 27-09-2018.}

DOI : 10.5530/ijcep.2018.5.3.9

\section{Copyright}

(C) 2018 Phcog.Net. This is an openaccess article distributed under the terms of the Creative Commons Attribution 4.0 International license.

\begin{abstract}
Background and Aim: Hypertension has been reported to be caused by sympathovaga imbalance and is associated with a wide variety of cognitive loss. The aim of present study is to study the association of sympathovagal imbalance with cognitive deficit, insulin resistance and oxidative stress in newly diagnosed hypertensive subjects. Methods: 84 subjects (42 normotensives and 42 hypertensives) aged between 18-44 years were included in this case-control study. The demographic, anthropometric, basal parameters, heart rate variability (HRV), cardiovascular autonomic function tests (CAFTs), event-related potential P300 and biochemical parameters were recorded. Association of various factors with cognitive deficit was studied by Pearson correlation analysis and independent contribution of factors to cognitive deficit was assessed by multiple regression analysis. Results: LF-HF ratio was increased and total power of HRV was reduced in hypertensives. Among CAFTs, 30:15 ratio and $\mathrm{DDBP}_{\text {G }}$ group were increased; $\mathrm{E}$ : I ratio was decreased in hypertensive group. The latency of P300 was significantly prolonged in hypertensives and P300 latency was positively correlated and linked with LF: HF ratio (the marker of SVI) in hypertensives. The fasting blood glucose, insulin, HOMA-IR, hsCRP and total oxidant capacity were increased and antioxidant status was decreased in hypertensive groups. Conclusion: Newly diagnosed hypertensive subjects have sympathovagal imbalance and considerable memory impairment even before medical intervention. Insulin resistance and oxidative stress contribute to the memory impairment and sympathovagal imbalance could be the physiological link for cognitive loss in newly diagnosed hypertensive subjects
\end{abstract}

Key words: Newly diagnosed hypertension, Sympathovagal imbalance, Cognitive deficit, Insulin resistance, Oxidative stress.

\section{INTRODUCTION}

According to $7^{\text {th }}$ Joint National Committee (JNC-7) criteria, hypertension is defined as systolic blood pressure $\geq 140 \mathrm{mmHg}$ and diastolic blood pressure $\geq 90 \mathrm{mmHg} \cdot{ }^{[1]}$ Globally, more than one billion adults above 25 years of age have hypertension which may rise to 1.56 billion by $2025 .{ }^{[2,3]}$ In developing countries like India, hypertension is the cause for $57 \%$ of all deaths from stroke and $24 \%$ of all deaths from coronary heart disease. ${ }^{[4]}$ High systolic blood pressure contributes to ischemic stroke and lacunar infarct, diffuse white matter damage or leukoaraiosis and this reduction in white-matter density possibly causes impaired cognitive functioning especially of executive functions. ${ }^{\left[{ }^{[]}\right.}$Hypertension, an established risk factor for cognitive deficit accounts for the third cause of death, out of one in eight deaths worldwide. ${ }^{[6,7]}$ Cognitive deficit is defined as an average cognitive performance at around the thirty-fifth to forty-fifth percentiles of normative data affecting one or multiple domains of cognition, though the cut-off to detect cognitive deficit is at fifth to tenth percentile. ${ }^{\left[{ }^{[3}\right.}$
Though there are various sophisticated techniques for determining cognitive impairment, recently the event related potential recorded in the form of positive wave at $300 \mathrm{~ms}$ (P300) has been considered as a better tool for assessing cognitive deficit. P300 is closely related to cognition-related brain functions like attention, intelligence and working memory as P300 activates multiple brain cortical areas which include frontal, prefrontal and parietal region..$^{[9-13]}$ Autonomic homeostasis contributes to higher brain functions including cognition. ${ }^{[14]}$

Recently we have reported from our laboratory that sympathovagal imbalance (SVI) caused by vagal inhibition in addition to the heightened sympathetic activity as the physiological basis of hypertension. ${ }^{[15,16]}$ Further, metabolic derangements which include dyslipidemia, insulin resistance and retrograde inflammation are contributors to sympathovagal imbalance in hypertension. ${ }^{[17,18]}$ However, there is paucity of data demonstrating the association of autonomic imbalance and metabolic derangements
Cite this article: Subathra TA, Pal GK, Dhanalakshmi Y, Pal P, Nanda N. Association of Sympathovagal Imbalance with Cognitive Deficit, Insulin Resistance and Oxidative Stress in Newly Diagnosed Hypertension. Int J Clin Exp Physiol. 2018;5(3):145-50 
with cognitive impairment in newly diagnosed hypertensive subjects in Indian population. Also, in previous studies assessing memory impairment in hypertension, cognitive functions have been assessed subjectively in hypertension and not objectively. Therefore, the present study was designed to assess the association of sympathovagal imbalance, insulin resistance and oxidative stress with cognitive deficit in patients with hypertension.

\section{METHODS}

This case-control study was conducted after receiving approval from the JIPMER Scientific Advisory Committee and Institute Ethics Committee for human studies. This study was conducted in the Autonomic Function Testing Laboratory and Electrophysiology Laboratory, Department of Physiology, JIPMER. The subjects were recruited from medicine outpatient department of JIPMER.

\section{Sample size calculation}

Total sample size was calculated to be 84 subjects ( 42 control subjects and 42 hypertensive subjects). The objective of this study was to measure and compare the LF-HF ratio with P300. Therefore, using previous reference, considering the mean and standard deviation values of LF-HF ratio, accepting power as $80 \%$ and keeping the level of significance $5 \%$, ${ }^{[19]}$ the total sample size calculated by Open Epi software was 84 .

\section{Estimation of biochemical parameters}

Written informed consent was obtained from all the subjects prior to commencement of the study. From each subject, $5 \mathrm{ml}$ of fasting blood sample was collected and fasting blood glucose was estimated by oxidation - reduction method using glucometer (Accu-chek Performa, Roche diagnostics; Sweden). The serum insulin was assayed using ELISA kit from Chemux BioScience Inc, CA and HOMA-IR is computed from the formula, HOMA-IR = fasting serum insulin $(\mu \mathrm{U} / \mathrm{ml}) \times$ fasting blood glucose $(\mathrm{mg} / \mathrm{dl}) / 405$. The inflammatory marker hsCRP was assayed using ELISA kit from Calbiotech Inc., CA, oxidative stress markers was assayed using QuantiChromTM TBARS assay kit from bioassay systems, CA to detect oxidant status and QuantiChromTM Antioxidant assay kit from bioassay systems, CA to detect antioxidant status.

\section{Inclusion criteria:}

Based on Joint National Committee -7 classification (1) the subjects were divided into:

Normotensives $(n=42)$ : systolic blood pressure $100-119 \mathrm{mmHg}$ and diastolic blood pressure 60-79 $\mathrm{mmHg}$, healthy person.

Hypertensives $(n=42)$ : Newly diagnosed hypertensives, having systolic blood pressure of $140 \mathrm{mmHg}$ or above and diastolic blood pressure of $90 \mathrm{mmHg}$ or above.

\section{Exclusion criteria:}

1. Subjects receiving any medications or having any acute illness.

2. History of smoking, alcoholism, endocrinal disorder, cardiovascular disese, neurological disorders and renal disorders.

3. Subjects practicing regular sports / athletic activities.

\section{Recording of blood pressure}

Subjects were asked to report to autonomic function laboratory of Physiology Department at about 9 AM following a light breakfast, without tea or coffee. After obtaining the informed consent, their age, height, body weight and body mass index (BMI) were recorded. Omron (SEM 1 Model), the automatic BP monitor (Omron Healthcare Co. Ltd, Kyoto, Japan) was used to measure BP. The cuff size of the equipment was
$121 \mathrm{~mm}$ (width) $\times 446 \mathrm{~mm}$ (length) and cuff tube length was $600 \mathrm{~mm}$. The subjects were asked to sit upright with back straight on a wooden armed chair and instructed to rest one forearm on a wooden table comfortably. They were asked to rest their other forearm on the hand rest of the chair. The height of the table was adjusted to an extent; the middle of the arm of the subjects when placed on the table coincided with the level of the heart. The blood pressure cuff was tied on the arm approximately $2 \mathrm{~cm}$ above the cubital fossa and care was taken that the cuff was neither too tight nor loose. For each participant, systolic blood pressure, diastolic blood pressure, heart rate were recorded at an interval of $5 \mathrm{mins}$ in each arm twice and the mean of the 4 recordings was considered for each parameter.

\section{Recording of heart rate variability (HRV)}

After 15 mins of supine rest, ECG was recorded for 5 mins for short-term HRV analysis following the guidelines of Task Force. ${ }^{[20]}$ ECG electrodes were connected and Lead II ECG was acquired at 500 samples per second for each channel using PowerLab 8/30 ML 870 data acquisition system with Lab chart pro software. The acquired 5 min resting lead II ECG (filtered with band pass filters) was carefully analyzed for ectopic and artifacts, which were meticulously removed manually. The detection of R-waves was done with a thresholding algorithm of Lab chart pro software. From the RR tachogram, power spectral analysis was performed by fast-fourier transform. Time-domain parameters (RMSSD, SDNN, NN50 and pNN50) and frequency domain parameters [total power (TP) of HRV, normalized LF power (LFnu), normalized HF power (HFnu), ratio of low-frequency to high-frequency power (LF-HF ratio)] were computed using HRV analysis software (Kubios HRV, version 2.2 Finland).

\section{Recording of P300 event-related potential}

Cognitive event-related potential (P300, positive wave at $300 \mathrm{~ms}$ ) was recorded in electrophysiology laboratory of Department of Physiology, JIPMER using Nihon Kohden Electrophysiology / electromyography (EP/EMG) machine. The recommendation of the International Federation of Clinical Neurophysiology was used ${ }^{[21]}$ and as per protocol of recording in Indian laboratory set-up. ${ }^{[22]}$ The subjects were instructed to come with cleaned oil-free scalp (shampoo head bath) and without ear wax before recording. They were asked to relax for $10 \mathrm{mins}$ and the procedure of recording was explained to them in detail. The scalp of the subject was cleaned with spirit and the electrode placements were done according to the 10-20 international system of EEG. The active, reference and ground electrodes were connected to channel 1 preamplifier with an impedance of $\leq 2 \mathrm{k} \Omega$. The midpoint between both the tragus and the midpoint between nasion and occipital protuberance were marked. At the point of intersection of above midpoints active recording electrode $\mathrm{Cz}$ (central zero point on scalp) was placed. With the help of jumper electrode, reference electrodes were placed one on the two mastoids. The ground electrode was placed in forehead Fz near to the hairline. The electrodes used were made of $\mathrm{Ag}-\mathrm{AgCl}$. $\mathrm{P} 300$ was recorded in the context of a standard auditory oddball paradigm. The band pass filter range was kept at $0.1 \mathrm{~Hz}$ and $50 \mathrm{~Hz}$. The auditory stimulus was given binaurally through a headphone. The subjects were asked to relax totally and asked to concentrate on the rare stimulus. The stimuli were given with the intensity of $40 \mathrm{~dB}$ with the 'tone' as the target or rare stimulus and 'click' as non-target or frequent stimulus. The stimulus frequency for tone burst and click were $2000 \mathrm{~Hz}$ and $1000 \mathrm{~Hz}$ respectively. The click duration was kept at $0.1 \mathrm{~ms}$. The stimulus occurrence speed was 1 stimulus per second. Only investigator and the subject were present in the laboratory and total silence was ensured during the recording. The participants were asked to open their eyes and fix to a point to avoid alpha waves in EEG. The rare stimuli were applied randomly and the percentage of rare stimuli was set at $20 \%$ and 
frequent stimuli at $80 \%$ of random. The stimulation rate was $0.5 \mathrm{~Hz}$ per second. The number of stimuli to be given was preset at 30 . The signals were picked by electrodes, filtered, amplified, averaged, displayed and analyzed using Neuropack software on the screen of Nihon Khoden EP/ EMG machine.

$\mathrm{N} 1$ was the negative wave at $100 \mathrm{~ms}, \mathrm{~N} 2$ was the negative wave at $200 \mathrm{~ms}$, P2 was the positive wave at $200 \mathrm{~ms}$ and P3 was the positive wave at $300 \mathrm{~ms}$. Among these waves, P300 i.e., the positive wave at $300 \mathrm{~ms}$, was considered as the marker of cognition. The procedure of recording was repeated for reproducibility of $\mathrm{P} 300$ and the marking was done for the latencies of N1, P2, N2 and P300 in milliseconds and the amplitudes of N1-P2, P2-N2 and N2-P3 in microvolts.

\section{Statistical analysis of data}

Statistical analysis was performed by SPSS version 19 (SPSS; SPSS Inc., Chicago, IL) for Windows. The data were subjected to KolmogorovSmirnov normality test. All the data were expressed as mean \pm SD. The intergroup differences in mean between the controls, prehypertensives and hypertensives were compared using one-way ANOVA and posthoc analysis was done using Tukey-Krammer for normally distributed data. The association of P300 with cardiovascular and biochemical parameters was assessed by Pearson's correlation analysis. Multiple regression analysis was performed to assess the independent association of P300 with different variables in hypertensives after adjusting for BMI and gender.

\section{RESULTS}

No significant difference was observed in age $(P=0.1007)$ in control and hypertensive group (Table 1 ). BMI and heart rate were significantly increased in hypertensives compared to controls (Table 1). On analysis of the frequency domain and time domain parameters of short term HRV, TP was significantly reduced among the hypertensives $(P<0.0001)$ compared to the controls. When the absolute powers were expressed in normalized units, LFnu was significantly elevated and HFnu was depressed in hypertensives $(P<0.0001)$ when compared to controls. The LF-HF ratio was significantly elevated in hypertensives $(P<0.0001)$ compared to controls (Table 1). The analysis of the time domain parameters revealed that there was a highly significant decrease in RMSSD $(P<0.0001)$ and SDNN $(P<0.0001)$ among hypertensives compared to controls. pNN50 $(P<0.0001)$ and NN50 $(P<0.0001)$ were significantly reduced in hypertensive group compared to controls (Table 1 ).

There was a significant reduction in E: I ratio $(P<0.005)$ and a significant increase in $30: 15$ ratio $(P<0.0001)$ and $\triangle \mathrm{DBP}_{\mathrm{IHG}}$ during isometric handgrip in hypertensive group compared to control group. The P300 latency was significantly prolonged in hypertensives compared to controls (Table 2). Though the amplitude of P300 wave is decreased in hypertensive subjects the difference was not statistically significant. Hypertensives showed significantly elevated fasting blood sugar $(P=0.0004)$, insulin $(P<0.0001)$ and HOMA-IR $(P<0.0001)$ values compared to controls (Table 3 ). There was significant elevation of hsCRP and TBARS in hypertensive group compared to control group. The total antioxidant status was reduced in hypertensive group compared to controls.

Table 4 depicts the correlation of $\mathrm{P} 300$ with various important parameters like BMI, fasting blood sugar, Insulin, HOMA-IR, TBARS, hsCRP and LF-HF ratio of control and hypertensive groups. There was significant positive correlation with hypertensive group when compared to control group. Table 5 shows multiple regression analysis to assess the independent association of P300 (as dependent variable) with BMI, HOMA-IR, TBARS, hsCRP and LF-HF ratio (as independent variables) in hypertensive group. In the present study, there is significant independent contribution of HOMA-IR $(\beta=0.245, \mathrm{P}=0.040)$, TBARS $(\beta=0.266, \mathrm{P}=0.032)$, LF-HF ratio $(\beta=0.351, P=0.009)$.
Table 1: Comparison of demographic, anthropometric, basal parameters, frequency domain indices (FDI) and time domain indices (TDI) of heart rate variability (HRV) and cardiac autonomic function tests (CAFT) parameters between control and hypertensive groups.

\begin{tabular}{lccc}
\multicolumn{1}{c}{ Parameters } & $\begin{array}{c}\text { Controls } \\
(\mathbf{n}=42)\end{array}$ & $\begin{array}{c}\text { Hypertensives } \\
(\mathbf{n}=42)\end{array}$ & $\boldsymbol{P}$ value \\
Age (years) & $30.19 \pm 5.01$ & $32.21 \pm 6.09$ & 0.1007 \\
Body weight $(\mathrm{kg})$ & $64.12 \pm 10.68$ & $71.82 \pm 6.27$ & 0.0001 \\
Height $(\mathrm{m})$ & $1.62 \pm 0.10$ & $1.64 \pm 0.09$ & 0.3382 \\
BMI $\left(\mathrm{Kg} / \mathrm{m}^{2}\right)$ & $24.34 \pm 2.81$ & $26.86 \pm 2.59$ & 0.0001 \\
BHR $(\mathrm{bpm})$ & $73.24 \pm 10.58$ & $85.21 \pm 9.70$ & 0.0001 \\
SBP (mmHg) & $111.88 \pm 7.98$ & $149.50 \pm 8.11$ & 0.0001 \\
DBP (mmHg) & $68.48 \pm 6.19$ & $99.31 \pm 6.48$ & 0.0001 \\
FDI parameters & & & \\
TP(ms $\left.{ }^{2}\right)$ & $1054.64 \pm 734.05$ & $553.55 \pm 300.51$ & 0.0001 \\
LFnu & $40.54 \pm 16.27$ & $63.07 \pm 12.07$ & 0.0001 \\
HFnu & $59.46 \pm 17.13$ & $36.92 \pm 2.07$ & 0.0001 \\
LF-HF ratio & $0.82 \pm 0.54$ & $1.99 \pm 0.96$ & 0.0001 \\
TDI parameters & & & \\
SDNN (ms) & $46.20 \pm 19.61$ & $24.06 \pm 8.04$ & 0.0001 \\
RMSSD (ms) & $64.53 \pm 24.67$ & $29.05 \pm 12.13$ & 0.0001 \\
NN50 & $50.35 \pm 21.08$ & $29.83 \pm 12.45$ & 0.0001 \\
pNN50 (\%) & $30.83 \pm 13.65$ & $17.04 \pm 5.84$ & 0.0001 \\
CAFT Parameters & & & \\
30:15 ratio & $1.21 \pm 0.29$ & $1.50 \pm 0.35$ & 0.0001 \\
E:I ratio & $1.39 \pm 0.17$ & $1.22 \pm 0.35$ & 0.0058 \\
DDBP IHG & $19.30 \pm 5.22$ & $28.19 \pm 6.45$ & 0.0001
\end{tabular}

Values expressed as mean $\pm \mathrm{SD}$; Analysis done by student's unpaired $t$ test. The $\mathrm{P}$ values less than 0.05 were considered statistically significant.

BMI: Body Mass Index; BHR: Basal heart rate; SBP: Systolic blood pressure; DBP: Diastolic blood pressure; TP: Total Power; LFnu: Low Frequency component expressed as normalized unit; HFnu: High Frequency component expressed as normalized unit; LF-HF ratio: ratio of low-frequency power to high-frequency power of heart rate variability; SDNN: Standard Deviation of Normal to Normal interval; RMSSD: Square root of the mean squared differences of successive normal to normal intervals; NN50: the number of interval differences of successive NN intervals greater than $50 \mathrm{~ms}$; pNN50: the proportion derived by dividing NN50 by the total number of NN intervals; 30:15 ratio: Ratio between maximum $\mathrm{RR}$ interval at $30^{\text {th }}$ beat and minimum RR interval at $15^{\text {th }}$ beat; E:I ratio: ratio of longest $\mathrm{RR}$ interval during expiration to the shortest $\mathrm{RR}$ interval during inspiration averaged over 6 cycles of respiration; $\triangle \mathrm{DBP}_{\mathrm{IHG}}$ : difference in diastolic blood pressure between supine and Isometric Hand Grip

\section{DISCUSSION}

In the present study, a significant prolongation of P300 latency in treatment naïve hypertensives (Table 2 ) indicates significant memory impairment in these subjects, as event-related potential is an established marker of higher cognitive function. Hypertension has been reported to be associated with a broad variety of cognitive loss including attention deficit, slowing of mental processing speed, impaired memory and reduced abstract reasoning. ${ }^{[23-27]}$ However, there are no reports from Indian subcontinent assessing the mechanisms of or the factors contributing to the cognitive loss in hypertension.

Previous studies have reported that the primary pathophysiology involved in elevated blood pressure in hypertensive subjects is the sympathovagal 
Table 2: Comparison of P300 latency and amplitude between control and hypertensive groups.

\begin{tabular}{lccc}
\multicolumn{1}{c}{ Parameters } & $\begin{array}{c}\text { Controls } \\
(\mathrm{n}=42)\end{array}$ & $\begin{array}{c}\text { Hypertensives } \\
(\mathrm{n}=42)\end{array}$ & P value \\
$\mathrm{N} 100(\mathrm{~ms})$ & $107.71 \pm 11.77$ & $121.07 \pm 31.40$ & 0.0116 \\
$\mathrm{P} 200(\mathrm{~ms})$ & $194.95 \pm 26.62$ & $204.90 \pm 28.25$ & 0.1005 \\
$\mathrm{~N} 200(\mathrm{~ms})$ & $231.76 \pm 25.70$ & $246.12 \pm 31.67$ & 0.0251 \\
$\mathrm{P} 300(\mathrm{~ms})$ & $341.57 \pm 44.56$ & $366.40 \pm 30.53$ & 0.0038 \\
$\mathrm{~N}_{1}-\mathrm{P}_{2} \mu \mathrm{v}$ & $8.07 \pm 5.75$ & $5.65 \pm 3.81$ & 0.0256 \\
$\mathrm{P}_{2}-\mathrm{N}_{2} \mu \mathrm{v}$ & $3.60 \pm 2.42$ & $3.04 \pm 2.67$ & 0.3168 \\
$\mathrm{~N}_{2}-\mathrm{P}_{3} \mu \mathrm{v}$ & $12.03 \pm 4.92$ & $10.98 \pm 2.57$ & 0.2237
\end{tabular}

Values expressed as mean $\pm \mathrm{SD}$; Analysis done by student's unpaired $t$ test. The $\mathrm{P}$ values less than 0.05 were considered statistically significant.

N100 (N1): Negative wave that appears in 100ms from application of stimulus in ERP tracing. P200 (P2): Positive wave that appears in $200 \mathrm{~ms}$ from application of stimulus in ERP tracing. N200 (N2): Negative wave that appears in 200ms from application of stimulus in ERP tracing. P300 (P3): Positive wave that appears in $300 \mathrm{~ms}$ from application of stimulus in ERP tracing.

Table 3: Comparison of biochemical parameters between control and hypertensive groups.

\begin{tabular}{lccc}
\multicolumn{1}{c}{ Parameters } & $\begin{array}{c}\text { Controls } \\
(\mathrm{n}=42)\end{array}$ & $\begin{array}{c}\text { Hypertensives } \\
(\mathrm{n}=42)\end{array}$ & P value \\
FBG $(\mathrm{mg} / \mathrm{dl})$ & $79.09 \pm 9.59$ & $86.29 \pm 8.32$ & 0.0004 \\
Insulin $(\mu \mathrm{U} / \mathrm{l})$ & $7.48 \pm 2.98$ & $21.40 \pm 4.63$ & 0.0001 \\
HOMA IR & $1.47 \pm 0.63$ & $4.34 \pm 3.29$ & 0.0001 \\
hsCRP $(\mathrm{mmol} / \mathrm{l})$ & $2.24 \pm 1.92$ & $11.81 \pm 6.42$ & 0.0001 \\
$\operatorname{TBARS}(\mu \mathrm{M})$ & $1.03 \pm 0.73$ & $2.29 \pm 1.06$ & 0.0001 \\
$\operatorname{TAS}(\mu \mathrm{M})$ & $419.23 \pm 130.68$ & $250.85 \pm 85.63$ & 0.0001
\end{tabular}

Values expressed as mean $\pm \mathrm{SD}$; Analysis done by student's unpaired $t$ test.

The $P$ values less than 0.05 were considered statistically significant.

FBG: Fasting blood glucose, HOMA- IR: Homeostasis model assessment of insulin resistance;

hsCRP: high-sensitivity C-reactive protein; TBARS: Thiobarbituric acid reactive substance; TAS: Total oxidant status.

imbalance. ${ }^{[16,17]}$ In the present study, LF-HF ratio, the indicator of sympathovagal imbalance, ${ }^{[28]}$ was significantly elevated in hypertensive groups (Table 1), indicating the presence of sympathovagal imbalance in treatment naïve hypertensives. The LF-HF ratio was significantly correlated with P300 in hypertensive groups, but not in control groups (Table 4), depicting the significant association of sympathovagal imbalance with cognitive impairment in hypertensives. Further, LH-HF ratio had independent contribution to P300 in hypertensives as demonstrated by multiple regression analysis (Table 5). Thus, findings of the present study indicate the close link of sympathovagal imbalance I with memory loss in hypertensive subjects.

All the time-domain indices of HRV (RMSSD, SDNN, NN50, pNN50) were significantly reduced in hypertensive groups compared to control group (Table 1) demonstrating that parasympathetic autonomic modulation was considerably less in these subjects as time-domain indices indicate cardiac vagal drive. ${ }^{[20,28]}$ Further, this was supplemented by reduced HFnu and TP of HRV (Table 1). As HFnu of HRV is the indicator of vagal drive to the heart and TP reflects overall power of vagal cardiac regulation. ${ }^{[2]}$ Thus, these findings represent decreased vagal drive of cardiac autonomic control in hypertensive subjects. Significant raise
Table 4: Correlation of $\mathrm{P} 300$ with various important parameters of control and hypertensive groups.

\begin{tabular}{lcccc}
\multicolumn{1}{c}{ Parameters } & \multicolumn{2}{c}{ Controls $(\mathrm{n}=42)$} & \multicolumn{2}{c}{ Hypertensives $(\mathrm{n}=42)$} \\
& $\boldsymbol{r}$ & $\boldsymbol{P}$ & $\boldsymbol{r}$ & $\boldsymbol{P}$ \\
BMI & 0.092 & 0.148 & 0.315 & 0.009 \\
FPG & 0.032 & 0.256 & 0.268 & 0.044 \\
Plasma insulin & 0.076 & 0.190 & 0.287 & 0.032 \\
HOMA-IR & 0.040 & 0.282 & 0.380 & 0.007 \\
TBARS & 0.030 & 0.257 & 0.298 & 0.013 \\
hsCRP & 0.080 & 0.162 & 0.260 & 0.046 \\
LF-HF ratio & 0.160 & 0.117 & 0.438 & 0.000
\end{tabular}

The $\mathrm{p}$ value $<0.05$ was considered significant. P300: Positive wave at $300 \mathrm{~ms}$ in event-related potential tracing; BMI: Body mass index; RPP: Rate pressure product; LF-HF ratio: ratio of low frequency to high frequency power of heart rate variability.

in 30:15 ratio and fall in E: I ratio in hypertensives (Table 1) indicates diminution in parasympathetic reactivity in these subjects, as these two parameters represent vagal reactivity. Significant increase in $\triangle \mathrm{DBP}_{\mathrm{IHG}}$ in hypertensives indicates increased sympathetic reactivity in these subjects, as $\triangle \mathrm{DBP}_{\mathrm{IHG}}$ represents sympathetic reactivity. ${ }^{[30]}$ Thus, autonomic imbalance in subjects with hypertension is due to the augmented sympathetic activity as well as reactivity and diminished vagal activity as well as reactivity.

There are reports of metabolic derangements in hypertension. ${ }^{[16]}$ There is also report of decline in cognitive function in conditions of metabolic derangements like dyslipidemia, IR and oxidative stress. ${ }^{[31-33]}$ However, there are no reports demonstrating cognitive impairment in hypertensives, especially in Indian population. In the present study, fasting blood sugar, insulin and HOMA-IR were significantly increased in hypertensives compared to normotensives (Table 3). Persistent hyperglycemia, advanced glycation end-products (AGEs) and hyperinsulinemia have been implicated in the genesis of memory loss, brain aging and Alzheimer's disease. ${ }^{[31,32]}$ Insulin resistance has also been strongly implicated in the development of cognitive impairment in Alzheimer's disease. ${ }^{[33]}$ In the present study, levels of fasting blood glucose, insulin and HOMA-IR were not only significantly high in hypertensive patients compared to control subjects (Table 3), but also HOMA-IR was significantly correlated with P300 in these subjects (Table 4). Further, HOMA-IR had independent contribution to P300 in hypertensives as demonstrated by multiple regression analysis (Table 5). Fasting blood glucose and insulin were not included in the same regression model to avoid multicolinearity. Thus, it appears that hyperinsulinemia and insulin resisitance are closely linked to cognitive impairment in hypertensive patients. Recently we have reported the link of insulin resistance to sympathovagal imbalance in polycystic ovary syndrome and prehypertension and in salt-preferring individuals and first-degree relatives of diabetes patients. Also, in the present study, HOMA-IR was significantly correlated with LF-HF ratio in hypertensive group. Therefore, sympathovagal imbalance might be the pathophysiological link between IR and cognitive impairment in hypertension.

Another linking mechanism of cognitive impairment in hypertensive patients could be the oxidative stress, as level of TBARS was significantly increased in hypertensive group compared to control groups (Table 3), Further, TBARS was significantly correlated with P300 in hypertensive group (Table 4) and TBARS had significant independent contribution to P300 in hypertensives (Table 5). Hippocampal oxidative stress has been implicated in the cognitive deficit in experimental diabetes model in 
Table 5: Multiple regression analysis to assess the independent association of $\mathrm{P} 300$ (as dependable variable) with various parameters (as independent variables) in hypertensive group after adjusting for BMI and gender.

\begin{tabular}{lcccc}
\multicolumn{1}{c}{$\begin{array}{c}\text { Independent } \\
\text { variables }\end{array}$} & $\begin{array}{c}\text { Standardized } \\
\text { regression } \\
\text { coefficient beta }\end{array}$ & \multicolumn{2}{c}{$\begin{array}{c}\text { 95\% confidence } \\
\text { interval }\end{array}$} & P Values \\
& & $\begin{array}{c}\text { Upper } \\
\text { limit }\end{array}$ & $\begin{array}{c}\text { Lower } \\
\text { limit }\end{array}$ & \\
BMI $\left(\mathrm{Kg} / \mathrm{m}^{2}\right)$ & 0.178 & 0.007 & 0.292 & 0.108 \\
HOMA-IR & 0.245 & 0.003 & 1.380 & 0.040 \\
TBARS & 0.266 & 0.004 & 1.602 & 0.032 \\
hSCRP & 0.145 & 0.009 & 0.168 & 0.142 \\
LF-HF ratio & 0.351 & 0.017 & 2.275 & 0.009
\end{tabular}

The $p$ value $<0.05$ was considered significant. P300: Positive wave at $300 \mathrm{~ms}$ in event-related potential tracing; BMI: Body mass index; LF-HF ratio: ratio of low-frequency to high-frequency power of heart rate variability.

mice. Therefore, oxidative stress could be a plausible mechanism of cognitive impairment in hypertensive patients too as fasting blood glucose was also significantly more in these patients. Recently, oxidative stress has been linked to autonomic imbalance and we have reported the association of oxidative stress with sympathovagal imbalance in hypertension. ${ }^{[19]}$ Further, in the present study, TBARS was significantly correlated with LF-HF ratio in hypertensive group (Table 4). Also, total antioxidant status was considerably reduced in hypertensives. Therefore, it appears that the sympathovagal imbalance could be the pathophysiological link between oxidative stress and cognitive deficit in hypertensive patients. Though hsCRP, the nonspecific marker of inflammation was significantly more in hypertensives, it was not correlated with P300 in these subjects. Therefore, retrograde inflammation is unlikely to contribute to the memory loss in these subjects.

In the present study, high BMI in hypertensive group could be suggested as a potential cause of memory impairment, as obesity has been observed to be associated with memory loss. ${ }^{[29,34]}$ Though BMI was significantly correlated with P300 in hypertensive group (Table 4), the independent contribution of BMI to P300 was not significant (Table 5). Thus, it is unlikely that high BMI would have contributed to memory loss in hypertensive patients. Moreover, there is also report that association of cognitive impairment with BMI is not significant. ${ }^{[35]}$

Though the exact cause of cognitive loss is not known, few reports have suggested that cognitive decline in hypertension could be due to cerebrovascular damage. ${ }^{[36]}$ The connection of the frontal lobes to other cortical and subcortical structures such as limbic system is disrupted in white matter tract injuries that have profound influence on autonomic functions. ${ }^{[14]}$ However, the role of autonomic dysfunction affecting cognition has not been studied in hypertension.

As hypertension is known to cause cerebrovascular disease, ${ }^{[37]}$ and reports indicate the role of brain infarction and white matter disease in cognitive impairment, ${ }^{[38,39]}$ cerebrovascular mechanisms have been proposed for cognitive impairment in hypertensives. Among the non-cerebrovascular mechanisms, hyperinsulinemia, insulin resistance, oxidative stress and deposition of AGEs and amyloid $\beta$ proteins in the brain substance have been proposed as the mechanisms of cognitive deficit in diabetes. ${ }^{[40,41]}$ Further, as practice of yoga has been reported to improve cerebral blood flow $^{[42]}$ and restore sympathovagal balance, ${ }^{[43]}$ role of yoga in the improvement of cognitive functions in hypertensive patients should also be investigated.

The novelty of the present study is that this is the first report linking sympathovagal imbalance with cognitive deficit in treatment naïve hypertensives from Indian subcontinent. This is also the first report from Indian subcontinent assessing the contribution of insulin resistance and oxidative stress to cognitive impairment and the association of IR and oxidative stress with sympathovagal imbalance in hypertensive patients before initiation of treatment. The findings of the present study demonstrate that even before the hypertension was diagnosed clinically, these patients have considerable memory impairment. As such in developing countries like India where detection of hypertension can occur late and compliance to treatment is not very effective, cognitive loss in patients suffering from chronic disease like hypertension, especially in younger population could pose serious threat to socioeconomic development.

\section{Limitations of the Study}

Though the limitations of the present study are that we have not estimated amyloid $\beta$ proteins and not done brain imaging investigations in hypertensive patients, sympathovagal imbalance could be the physiological basis of cognitive impairment in hypertensives as chronic sympathovagal imbalance with sympathetic accentuation has been reported to cause hypertrophy of vascular wall with narrowing of vessel lumen causing decreased cerebral perfusion that might contribute to the cerebrovascular component of cognitive impairment. ${ }^{[41]}$ In the present study there is not a single hypertensive subject that does not appear to have metabolic alterations and therefore, a group of non-metabolic disease patients should have been included to eliminate the impact of metabolic alterations on cognitive deficit, which is a major limitation of the study. Further, other measures of cognitive assessment such as memory function tests through questionnaires should have been included in the study protocol.

\section{CONCLUSION}

Newly diagnosed hypertensive subjects have sympathovagal imbalance and considerable memory impairment even before medical intervention. Insulin resistance and oxidative stress contribute to the memory impairment and sympathovagal imbalance could be the physiological link for cognitive loss in newly diagnosed hypertensive subjects.

\section{ACKNOWLEDGEMENT}

There is no acknowledgement in this study.

\section{CONFLICT OF INTEREST}

The authors declare no conflicts of interest.

\section{ABBREVIATIONS USED}

FBG: Fasting blood glucose; HOMA- IR: Homeostasis model assessment of insulin resistance; hsCRP: high-sensitivity C-reactive protein; TBARS: Thiobarbituric acid reactive substance; TAS: Total oxidant status.

\section{SUMMARY}

Hypertension has been reported to be caused by sympathovagal imbalance and is associated with a wide variety of cognitive loss. The aim of present study is to study the association of sympathovagal imbalance with cognitive deficit, insulin resistance and oxidative stress in newly diagnosed hypertensive subjects. Eighty four subjects (42 normotensives and 42 hypertensives) aged between 18-44 years were included in this case-control study. The demographic, anthropometric, basal parameters, heart rate variability (HRV), cardiovascular autonomic function tests (CAFTs), event-related potential P300 and biochemical parameters were recorded. Association of various factors with cognitive deficit was studied by Pearson correlation analysis and independent contribution 
of factors to cognitive deficit was assessed by multiple regression analysis. LF-HF ratio was increased and total power of HRV was reduced in hypertensives. Among CAFTs, 30:15 ratio and DDBPIHG group were increased; E: I ratio was decreased in hypertensive group. The latency of P300 was significantly prolonged in hypertensives and P300 latency was positively correlated and linked with LF: HF ratio (the marker of SVI) in hypertensives. The fasting blood glucose, insulin, HOMA-IR, hsCRP and total oxidant capacity were increased and antioxidant status was decreased in hypertensive groups. Newly diagnosed hypertensive subjects have sympathovagal imbalance and considerable memory impairment even before medical intervention. Insulin resistance and oxidative stress contribute to the memory impairment and sympathovagal imbalance could be the physiological link for cognitive loss in newly diagnosed hypertensive subjects.

\section{REFERENCES}

1. Chobanian AV, Bakris GL, Black HR, Cushman WC, Green LA, Izzo JL, et al. Seventh report of the Joint National Committee on Prevention, Detection, Evaluation and Treatment of High Blood Pressure. Hypertens Dallas Tex. 2003;42(6):1206-52

2. Kearney PM, Whelton M, Reynolds K, Muntner P, Whelton PK, He J. Global burden of hypertension: analysis of worldwide data. Lancet Lond Engl. 2005;365(9455):217-23.

3. Greenlund KJ, Croft JB, Mensah GA. Prevalence of heart disease and stroke risk factors in persons with prehypertension in the United States, 1999-2000. Arch Intern Med. 2004;164(19):2113-8.

4. Gupta R. Trends in hypertension epidemiology in India. J Hum Hypertens. 2004;18(2):73-8.

5. Meissner A. Hypertension and the Brain: A Risk Factor for More than Heart Disease. Cerebrovasc Dis Basel Switz. 2016;42(3-4):255-62.

6. Qiu C, Winblad B, Fratiglioni L. The age-dependent relation of blood pressure to cognitive function and dementia. Lancet Neurol. 2005;4(8):487-99.

7. Ryuno H, Kamide K, Gondo Y, Nakama C, Oguro R, Kabayama M, et al. Differences in the association between high blood pressure and cognitive functioning among the general Japanese population aged 70 and 80 years: The SONIC study. Hypertens Res Off J Jpn Soc Hypertens. 2016;39(7):557-63.

8. Biessels GJ, Reagan LP. Hippocampal insulin resistance and cognitive dysfunction Nat Rev Neurosci. 2015;16(11):660-71.

9. McEvoy LK, Smith ME, Gevins A. Dynamic cortical networks of verbal and spatial working memory: effects of memory load and task practice. Cereb Cortex. 1998;8(7):563-74.

10. Knight RT, Grabowecky MF, Scabini D. Role of human prefrontal cortex in attention control. Adv Neurol 1995;66:21-36.

11. Frodl-Bauch $T$, Bottlender R, Hegerl U. Neurochemical substrates and neuroanatomical generators of the event-related P300. Neuropsychobiology. 1999;40(2):86-94.

12. Li L, Gratton C, Yao D, Knight RT. Role of frontal and parietal cortices in the control of bottom-up and top-down attention in humans. Brain Res. 2010;1344:173-84

13. Lim SS, Vos T, Flaxman AD, Danaei G, Shibuya K, Adair-Rohani H, et al. A comparative risk assessment of burden of disease and injury attributable to 67 risk factors and risk factor clusters in 21 regions, 1990-2010: a systematic analysis for the Global Burden of Disease Study 2010. Lancet Lond Engl. 2012;380(9859):2224-60.

14. Pal GK. The limbic system. In: Textbook of Medical Physiology. Ahuja Publications, New Delhi. 2011;2:909-17.

15. Pal GK, Pal P, Nanda N, Amudharaj D, Adithan C. Cardiovascular dysfunctions and sympathovagal imbalance in hypertension and prehypertension: physiological perspectives. Future Cardiol. 2013;9(1):53-69.

16. Pal GK, Adithan C, Amudharaj D, Dutta TK, Pal P, Nandan PG, et al. Assessment of sympathovagal imbalance by spectral analysis of heart rate variability in prehypertensive and hypertensive patients in Indian population. Clin Exp Hypertens NY N 1993. 2011;33(7):478-83

17. Pal GK, Adithan C, Ananthanarayanan PH, Pal P, Nanda N, Durgadevi T, et al Effects of gender on sympathovagal imbalance, prehypertension status and cardiovascular risks in first-degree relatives of type 2 diabetics. Am J Hypertens. 2014;27(3):317-24
18. Pal GK, Pal P, Nanda N, Lalitha V, Dutta TK, Adithan C. Sympathovagal imbalance in young prehypertensives: importance of male-female difference. Am J Med Sci. 2013;345(1):10-7.

19. Pal GK, Adithan C, Ananthanarayanan PH, Pal P, Nanda N, Thiyagarajan D, et al. Association of sympathovagal imbalance with cardiovascular risks in young prehypertensives. Am J Cardiol. 2013;112(11):1757-62.

20. Heart rate variability: standards of measurement, physiological interpretation and clinical use. Task Force of the European Society of Cardiology and the North American Society of Pacing and Electrophysiology. Circulation. 1996;93(5):1043-65.

21. Heinze HJ, Münte TF, Kutas M, Butler SR, Näätänen R, Nuwer MR, et al. Cognitive event-related potentials. The International Federation of Clinical Neurophysiology. Electroencephalogr Clin Neurophysiol Suppl. 1999;52:91-5.

22. Kumar N, Sood S, Singh M, Beena, Sakshi. Effect of acute moderate exercise on cognitive event-related potentials n100, p200, n200 and interpeak latencies. Indian J Psychol Med. 2010;32(2):131-5.

23. Elias MF, Goodell AL, Dore GA. Hypertension and cognitive functioning: a perspective in historical context. Hypertens Dallas Tex 1979. 2012;60(2):260-8.

24. Waldstein SR, Manuck SB, Ryan CM, Muldoon MF. Neuropsychological correlates of hypertension: review and methodologic considerations. Psychol Bull. 1991;110(3):451-68.

25. Gorelick PB, Scuteri A, Black SE, Decarli C, Greenberg SM, ladecola C, et al. Vascular contributions to cognitive impairment and dementia: a statement for healthcare professionals from the American heart association/american stroke association. Stroke J Cereb Circ. 2011;42(9):2672-713.

26. Iadecola C. Hypertension and Dementia. Hypertension. 2014;64(1):3-5

27. Chen KHM, Henderson VW, Stolwyk RJ, Dennerstein L, Szoeke C. Prehypertension in midlife is associated with worse cognition a decade later in middleaged and older women. Age Ageing. 2015;44(3):439-45

28. Malliani A. Heart rate variability: from bench to bedside. Eur J Intern Med. 2005; 16(1):12-20

29. Cronk BB, Johnson DK, Burns JM. Alzheimer's Disease Neuroimaging Initiative. Body mass index and cognitive decline in mild cognitive impairment. Alzheimer Dis Assoc Disord 2010;24(2):126-30.

30. Pal GK, Pal P. Autonomic Function Tests. In: Textbook of Practical Physiology. $3^{\text {rd }}$ edition. Orient Blackswan. 2011;245-52.

31. Park CR. Cognitive effects of insulin in the central nervous system. Neurosci Biobehav Rev. 2001;25(4):311-23.

32. Cole GM, Frautschy SA. The role of insulin and neurotrophic factor signaling in brain aging and Alzheimer's Disease. Exp Gerontol. 2007;42(1-2):10-21.

33. Craft S. Insulin resistance and Alzheimer's disease pathogenesis: potential mechanisms and implications for treatment. Curr Alzheimer Res. 2007;4(2):147-52

34. Whitmer RA, Gunderson EP, Barrett-Connor E, Quesenberry CP, Yaffe K. Obesity in middle age and future risk of dementia: a 27 year longitudinal population based study. BMJ. 2005;330(7504):1360.

35. Cournot M, Marquié JC, Ansiau D, Martinaud C, Fonds H, Ferrières J, et al. Relation between body mass index and cognitive function in healthy middleaged men and women. Neurology. 2006;67(7):1208-14.

36. Uiterwijk R, Huijts $M$, Staals J, Duits A, Gronenschild E, Kroon AA, et al Subjective cognitive failures in patients with hypertension are related to cognitive performance and cerebral microbleeds. Hypertens Dallas Tex 1979 2014;64(3):653-7.

37. Boden-Albala B, Cammack S, Chong J, Wang C, Wright C, Rundek T, et al. Diabetes, fasting glucose levels and risk of ischemic stroke and vascular events: findings from the Northern Manhattan Study (NOMAS). Diabetes Care. 2008;31(6):1132-7.

38. Vermeer SE, Prins ND, den Heijer T, Hofman A, Koudstaal PJ, Breteler MMB. Silent brain infarcts and the risk of dementia and cognitive decline. $N$ Engl J Med. 2003;348(13):1215-22

39. Pantoni L. White matter ischemia: Time to begin integrating experimental and clinical data. Eur Neurol. 2006;56(2):71-3.

40. VijayakumarTM, Sirisha GBN, Farzana BMD, Dhanaraju MD. Mechanism Linking Cognitive Impairment and Diabetes mellitus. Eur J Appl Sci. 2012;4(1):1-5.

41. Luchsinger JA. Type 2 diabetes and cognitive impairment: linking mechanisms. J Alzheimers Dis JAD. 2012;30(Suppl 2):S185-198.

42. Cohen DL, Wintering N, Tolles V, Townsend RR, Farrar JT, Galantino ML, et al. Cerebral blood flow effects of yoga training: preliminary evaluation of 4 cases. J Altern Complement Med NY N. 2009;15(1):9-14

43. Pal GK, Agarwal A, Karthik S, Pal P, Nanda N. Slow yogic breathing through right and left nostril influences sympathovagal balance, heart rate variability and cardiovascular risks in young adults. North Am J Med Sci. 2014;6(3):145-51. 